\title{
Original
}

\section{Immunolocalization of SP6, LEF1 and Associated Factors in the Tooth Germ of Rat Molars}

\author{
Mitsuko Moriguchi' ${ }^{1)}$, Mayu Kosika ${ }^{1)}$, Yasuo Miake ${ }^{1)}$, Yasuaki Yamaguchi ${ }^{2)}$, \\ Takaki Yamazaki ${ }^{1)}$ and Hitoshi Yamamoto ${ }^{1)}$
}

\author{
1) Department of Ultrastructural Science, Tokyo Dental College, Chiba, Japan. \\ 2) Department of Physical Therapy, School of Health Science, Niigata University of Health and Welfare, Niigata, Japan \\ (Accepted for publication, September 19, 2013)
}

\begin{abstract}
Lymphoid enhancer-binding factor 1 (LEF1) is a transcription factor in the Wnt/ $\beta$-catenin signaling pathway. LEF1 may control odontoblast differentiation. Specificity Protein 6/Epiprofin (SP6) is a transcription factor that mediates the signaling pathway between bone morphogenetic protein (BMP) and the Wnt/ $\beta$-catenin. LEF1 is decreased by the overexpression of SP6. In contrast, lef1 gene expression is increased in the dental epithelium and mesenchyme of SP6-deficient mice. Thus, LEF1 and SP6 act in an antagonistic manner, being involved in tooth formation. However, this has not been investigated in detail in vivo. In the present study, the expressions of Dickkopf-related protein-1 (DKK-1) (an antagonist to the Wnt signaling pathway), SP6, and phospho-Smad1/5/8 (p-Smad1/5/8) (activated R-Smad in the BMP signaling pathway) were immunohistochemically investigated using serial sections prepared from rat first molar tooth germs in the bell stage on embryonic day 19 and days 10 and 15 after birth, in order to investigate the roles of these factors in differentiation into ameloblasts and odontoblasts. As a result, p-Smad1/5/8 was strongly expressed in preodontoblasts and odontoblasts and also in the inner enamel epithelium and ameloblasts in the secretory stage, suggesting activation of the BMP signaling pathway through $\mathrm{p}$-Smad1/5/8 and involvement of the BMP signaling pathway in differentiation into odontoblasts and ameloblasts and their secretory functions. LEF1 appears in preodontoblasts and preameloblasts and disappears with differentiation, suggesting that it suppresses the differentiation into these cells. In contrast, SP6 and DKK1 expressions are enhanced with odontoblast differentiation. Therefore, the downregulation of the Wnt signaling pathway through SP6 and DKK1 is involved in the disappearance of LEF1 in odontoblasts. SP6 expression was enhanced with ameloblast differentiation, suggesting that the activated BMP signaling pathway suppressed the expression of LEF1 through SP6, thus being involved in ameloblast differentiation.
\end{abstract}

Keywords: LEF1, SP6, p-Smad1/5/8, DKK1, developing molar.

\section{Introduction}

In the Wnt/ß-catenin signaling pathway, Wnt binding to its receptor facilitates the transport and accumulation of $ß$-catenin in the nucleus. Then, $B$-catenin binds to a transcription factor, LEF1/ $\mathrm{TCF}$, to induce gene expressions ${ }^{1,2)}$. During tooth development, Wnt-10a and $-10 \mathrm{~b}$ are expressed in the mesenchyme ${ }^{3)}$ and epithelium ${ }^{4}$, respectively, thus being involved in differentiation into odontoblasts and ameloblasts and their functions through $\beta-$ catenin $^{5}$. Particularly, Wn-10a is expressed in preodontoblasts and secretory odontoblasts, being involved in dentinogenesis and mineralization through dentin sialophosphoprotein (DSPP) $)^{3)}$. LEF1 acting downstream of the signaling pathway regulates DSPP, controlling terminal odontoblast differentiation in vitro ${ }^{6}$. Lef1 is

Correspondence to: Mitsuko Moriguchi, PhD. Department of Ultrastructural Science, Tokyo Dental College, 2-9-18, Misakicho, Chiyoda-ku, Tokyo 101-0061, Japan, Email: moriguti@tdc.ac.jp, Tel: +81-03-6380-9274 expressed in the inner enamel epithelium of early tooth germs and dental papilla cells and preodontoblasts immediately below the epithelium in $v i v o^{7}$. Subsequently, lef1 disappears and is not expressed in odontoblasts and ameloblasts ${ }^{8}$. Lef1 is involved in maintaining the precursor phenotype and is also employed as a marker of preameloblasts ${ }^{9}$. Specifically, Wnt upstream of the signaling pathway is expressed in differentiated cells, while downstream lef1 is not expressed. The mechanism of this phenomenon is unclear. Dickkopf (DKK) binds to LRP, the receptor of Wnt, to inhibit Wnt signaling ${ }^{10,11}$. Particularly, Dkk1 is expressed in preodontoblasts and odontoblasts to regulate Wnt signaling, thus being involved in dentition and dental hard tissue formation $^{12)}$.

SP6 (Epiprofin) promotes amelogenesis by regulating the expression of follistatin, an antagonist of the BMP signaling pathway $^{13)}$. The overexpression of Sp6 in preameloblasts increases 

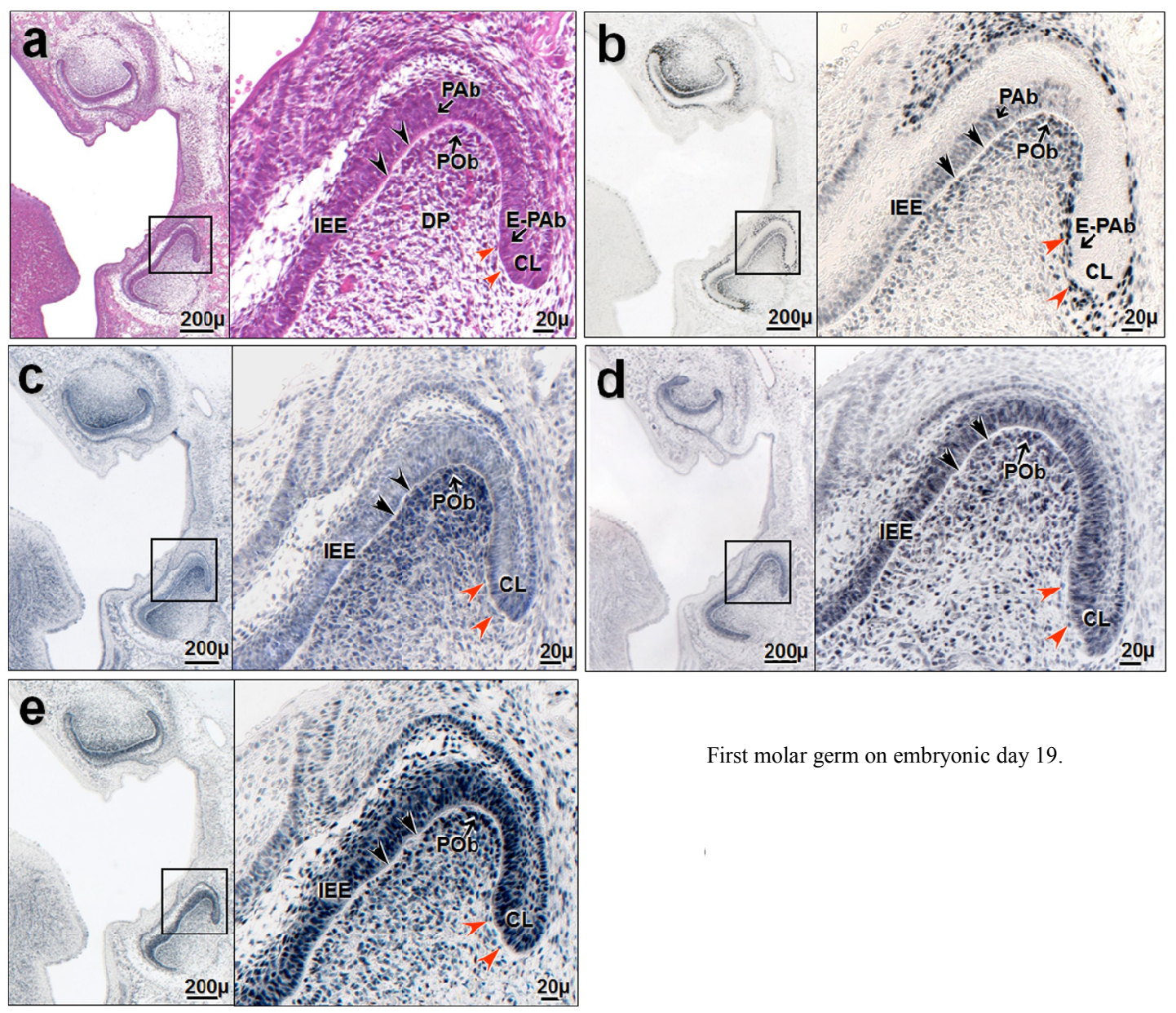

First molar germ on embryonic day 19.

Figure1. Immunohistochemical observation of the rat first molar germ on embryonic day 19.

a: Hematoxylin and eosin (H-E) staining. Predentin was slightly formed in the corner region of the dental papilla. Preameloblasts were observed in the inner enamel epithelium, while preodontoblasts were observed in the dental papilla, above and below the predentin. Around the cervical loop, cells corresponding to early preameloblasts were distributed in the inner enamel epithelium, while cells corresponding to early preodontoblasts were distributed in the dental papilla immediately below the epithelium. b: Immunostaining with antiLEF1. The reaction was positive in preameloblasts and negative in the early preameloblasts of the cervical loop. A strong positive reaction was observed in early preodontoblasts adjacent to the cervical loop. However, the reaction was attenuated in preodontoblasts. c: Staining with anti-DKK1 showed stronger reactions with preodontoblast differentiation from early preodontoblasts and was weak in the inner enamel epithelium. d,e: Staining with anti-SP6(d), or anti-P-Smad1/5/8(e) showed a uniform positive reaction in the inner enamel epithelium and an attenuated reaction with the transition from preodontoblasts to early preodontoblasts. The boxed areas are enlarged on the right of each panel. Black arrowheads Predentin, Red arrowhead early preodontoblast, POb Preodontoblast, DP Dental papilla, IEE Inner enamel epithelium, PAb Preameloblasts, E-PAb early preameloblasts, CL Cervical loop. Bars, $200 \mu \mathrm{m}$ or $20 \mu \mathrm{m}$

ameloblastin and decreases LEF1, a marker of preameloblasts. Therefore, SP6 promotes ameloblast differentiation from preameloblasts ${ }^{9}$. Sp6 is expressed in the fetal ectoderm under the control of the $\mathrm{Wnt} / \beta$-catenin signaling pathway. The expression of BMP-4 is decreased in Sp6-deficient mice. Therefore, SP6 mediates the signaling pathway between $\mathrm{Wnt} / \beta$-catenin and $\mathrm{BMP}^{14)}$. Sp6 appears in preodontoblasts and differentiating odontoblasts as well as the inner enamel epithelium, preameloblasts, and ameloblasts in vivo ${ }^{15)}$. No detailed report has been published on SP6 in odontoblasts. In the mandibular mesenchyme of Sp6-deficient mice, Lef1 is expressed ${ }^{9)}$ and Bmp and Dkk1 are decreased ${ }^{16)}$, suggesting a signaling pathway linking
LEF1 and BMP by SP6 through DKK1 ${ }^{16)}$. So, SP6 is expressed in both ameloblasts and odontoblasts to mediate the signaling pathways of both $\mathrm{Wnt} / \beta$-catenin and BMP, controlling the differentiation and functions of these cells. However, this has not been investigated in detail. We immunohistochemically investigated the distributions of signaling molecules to examine their interrelationships in the rat molar tooth germs in the early bell stage (i.e., just before differentiation into these cells) and in the late bell stage (i.e., during hard tissue formation) using antibodies against Dkk1 which inhibits the upstream Wnt/ $\beta$ catenin signaling pathway, LEF1 that functions downstream of this pathway, SP6 that mediates the signaling pathway between 

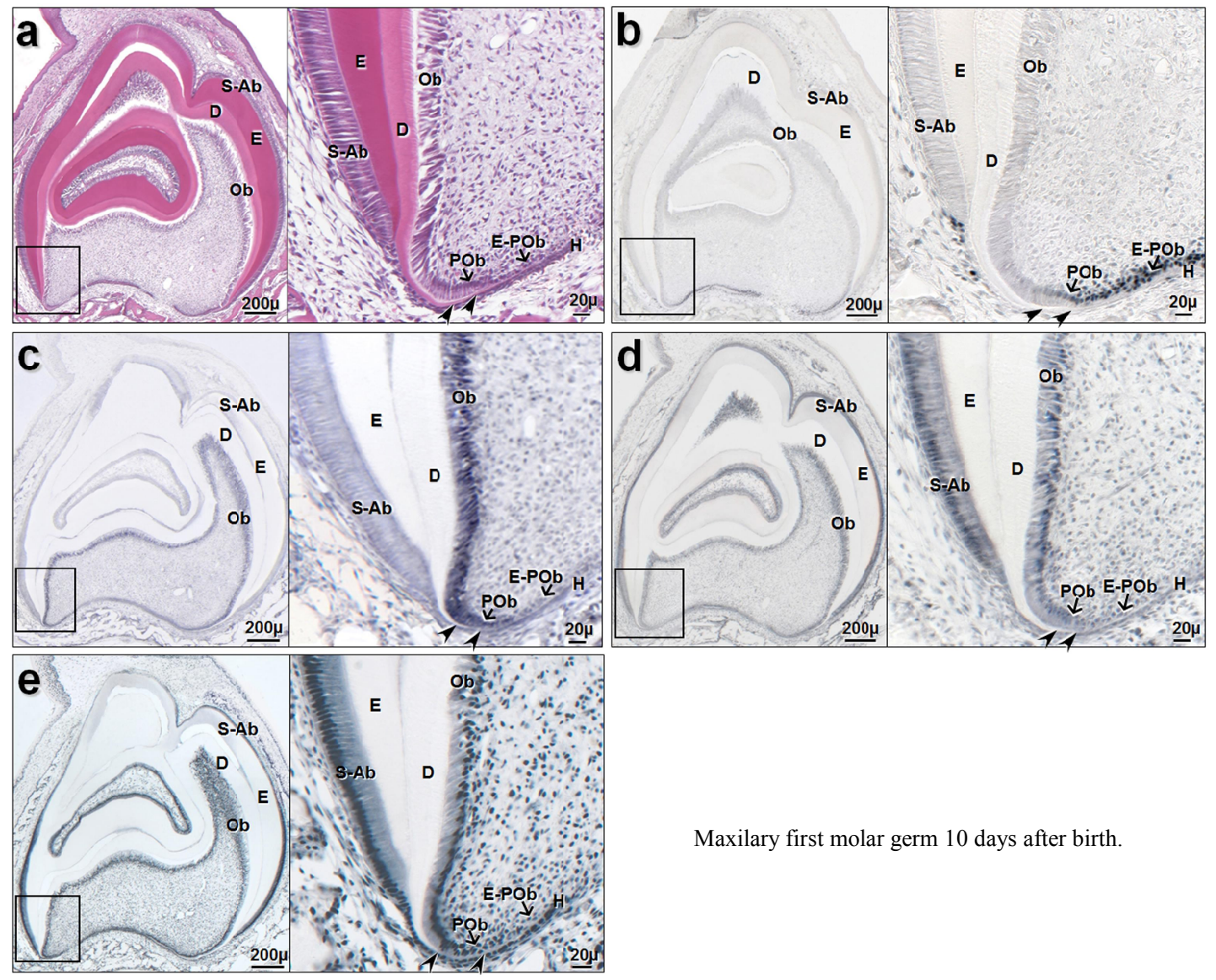

Maxilary first molar germ 10 days after birth.

Figure 2. Immunohistochemical observation of the rat maxillary first molar germ 10 days after birth.

a: H-E staining. At the edge of tooth neck formation, secretory ameloblasts, odontoblasts, preodontoblasts adjacent to the predentin and early preodontoblasts adjacent to Hertwig's epithelial root sheath were observed. b: Immunostaining with anti-LEF1 showed a strong reaction in the nucleus of early preodontoblasts, an attenuated reaction in preodontoblasts, and an unclear reaction in ameloblasts and odontoblasts. c: Anti-DKK1 antibody yielded a strong positive reaction in odontoblasts and an attenuated reaction with the transition from odontoblasts to early preodontoblasts. d,e: Anti-SP6(d) antibody showed a strong positive reaction in secretory ameloblasts and odontoblasts, and anti-P-Smad1/5/8(e) antibody showed a strong positive reaction in secretory ameloblasts, odontoblasts and preodontoblasts. However, these antibodies yielded an attenuated reaction with the transition from odontoblasts to early preodontoblasts. The boxed areas are enlarged on the right of each panel. Arrowheads predentin, E enamel, D dentin, S-Ab secretory ameloblast, Ob odontoblast, POb preodontoblast, E-POb early preodontoblasts, H Hertwig's epithelial root sheath. Bars, $200 \mu \mathrm{m}$ or $20 \mu \mathrm{m}$.

Wnt/ $\beta$-catenin and BMP, and p-Smad1/5/8 (i.e., activated R-Smad in the BMP signaling pathway).

\section{Materials and Methods}

\section{Tissue preparation}

The experiment was performed using Sprague-Dawley (SD) rats embryonic day 19, 10 days and 15 days after birth, according to the Guidelines for the Handling of Experimental Animals of Tokyo Dental College.

The rats were fixed by perfusion or immersion in $0.01 \mathrm{M}$ periodic acid-0.075 M lysine-2\% paraformaldehyde (PLP). Samples were fixed and decalcified with triethyl-ammonium EDTA in $80 \%$ ethanol ${ }^{17}$. The first molar tooth germ with surrounding tissues was removed, embedded in paraffin, and longitudinal sections 5 $\mu \mathrm{m}$ in thickness were prepared. Some of the sections were treated with hematoxylin and eosin (H-E) staining.

\section{Immunostaining}

The paraffin sections were deparaffinized, and the antigen was activated and unmasked by irradiating the sections twice with 400$\mathrm{W}$ microwaves in $0.01 \%$ citric acid buffer ( $\mathrm{pH} 6.0$ ) for $15 \mathrm{~min}$. Free aldehyde radicals were removed by immersing the sections in $0.1 \%$ glycine PBS for $30 \mathrm{~min}$, and the endogenous peroxidase activity was eliminated by immersion in $0.3 \% \mathrm{H}_{2} \mathrm{O}_{2}, 0.1 \% \mathrm{NaN}_{3}$, and PBS for $30 \mathrm{~min}$. Blocking was performed for $1 \mathrm{~h}$ with normal goat serum (1:10). The sections were incubated overnight at room temperature with the following diluted primary antibodies in $1 \%$ bovine serum albumin (BSA) PBS: LEF1(C12A5) (anti-LEF1, rabbit monoclonal antibody; Cell Signaling Technology, Danvers, MA; 1:500), DKK1 (EPR4759) (anti-DKK1, rabbit monoclonal antibody; Abcam, Cambridge, England; 1:300), SP6(anti-SP6 Cterminal amino acids 327-376, rabbit polyclonal antibody; Abcam, Cambridge, England; 1:100), and pSmad1/5/8(Phospho- 

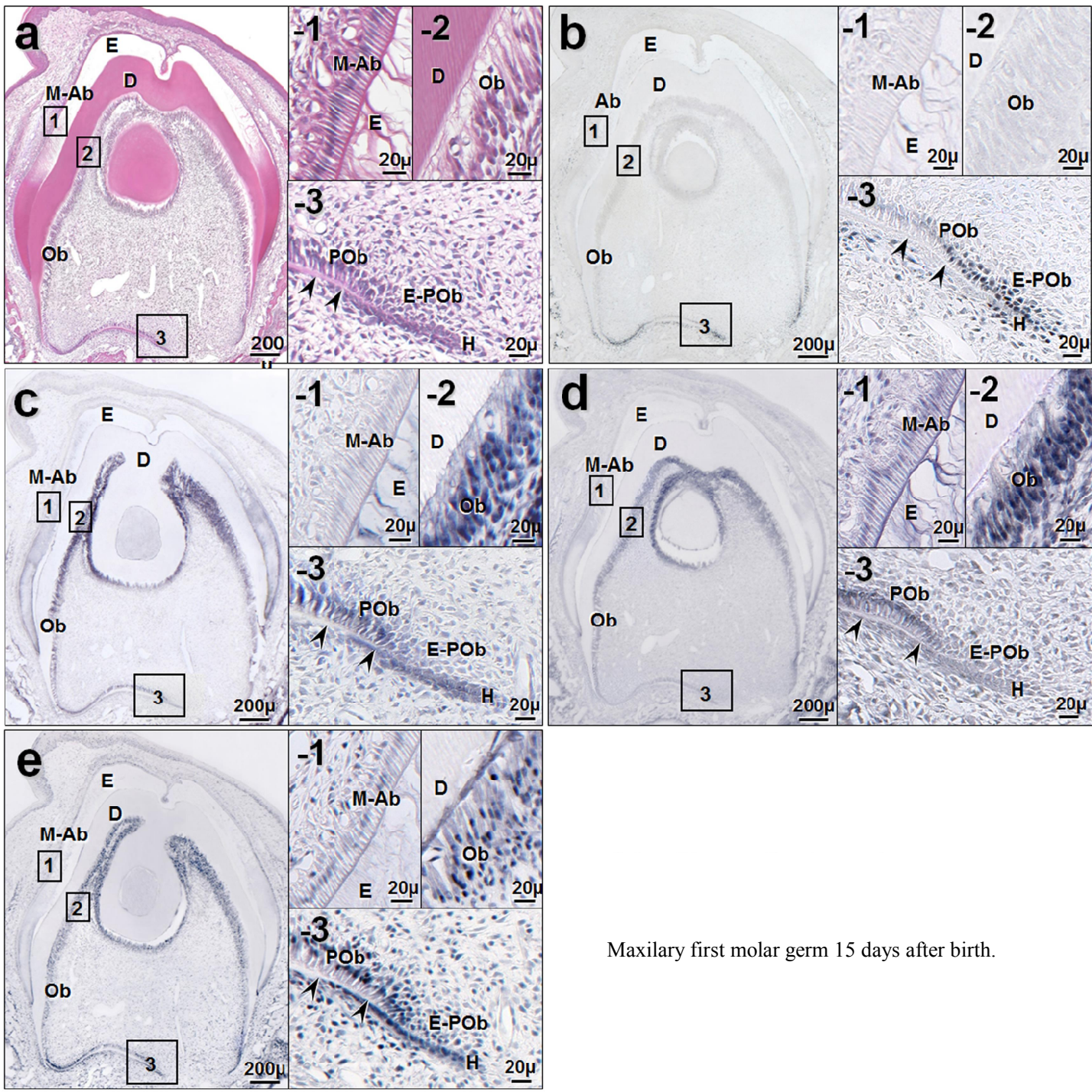

Maxilary first molar germ 15 days after birth.

Figure 3. Immunohistochemical observation of the rat maxillary first molar germ 15 days after birth.

a: H-E staining. Mature ameloblasts and odontoblasts were observed around the cusp. Preodontoblasts were observed along predentin at the apical end of root formation. Early preodontoblasts were observed along Hertwig's epithelial root sheath. b: Immunostaining with anti-LEF1 showed a strong positive reaction in the nuclei of early preodontoblasts but no reaction in odontoblasts or mature ameloblasts. The positive reaction was attenuated with odontoblast differentiation from early preodontoblasts. c, d, e: Anti-DKK1(c), anti-SP6(d) and anti-P-Smad1/5/8(e) antibodies showed a strong reaction in odontoblasts. These reactions were enhanced with odontoblast differentiation from early preodontoblasts. Mature ameloblasts were positive for SP6 and P-Smad1/5/8 but unclear for DKK1. The boxed areas $-1,-2$ and -3 are enlarged in figures $-1,-2$ and -3 of each panel. E enamel, D dentin, $\mathrm{M}-\mathrm{Ab}$ mature ameloblast, Ob odontoblast, POb preodontoblast, E-POb early preodontoblasts, H Hertwig's epithelial root sheath, Arrowheads predentin. Bars, $200 \mu \mathrm{m}$ or $20 \mu \mathrm{m}$.

Smad1(Ser463/465)/Smad5 (Ser463/465)/Smad8(Ser426/428) (anti-Phospho-Smad1/5/8, Phosphopeptide-Ser463/465 of Smad5, rabbit polyclonal antibody, Cell Signaling Technology; Danvers, MA; 1:100). Samples were then treated with a secondary antibody, biotin-labeled rabbit-anti-IgG (Elite ABC kit: VECTASTAIN) and avidin-biotinylated peroxidase complex (ABC) reagent (Elite ABC kit: VECTASTAIN) each at room temperature for $30 \mathrm{~min}$. In addition, color was developed with a $\mathrm{Ni}^{2+}$ $\mathrm{Co}^{2+}$-DAB solution18). For observations related to antibody reactions, negative control sections were produced by treating the sections with rabbit-IgG (1:500, 1:300 and 1:100).

\section{Results}

\section{Rat first molar germ on embryonic day 19 (early bell stage)}

Predentin was slightly formed in the corner region of the dental papilla. Preameloblasts were observed in the inner enamel epithelium, while preodontoblasts were observed in the dental papilla, above and below the predentin. Around the cervical loop, cells corresponding to early preameloblasts were distributed in the inner enamel epithelium, while cells corresponding to early 
Mitsuko Moriguchi et al.: LEF1, SP6, p-Smad1/5/8 and DKK1 in Developing Molars

Table 1. Immunohistochemical distribution of LEF1, DKK1, SP6 and P-Smad1/5/8 during rat tooth development.

\begin{tabular}{|c|c|c|c|c|c|}
\hline & & LEF1 & DKK1 & SP6 & $\begin{array}{l}\text { P-Smad } \\
1 / 5 / 8 \\
\end{array}$ \\
\hline \multirow{4}{*}{$\begin{array}{l}\text { Early bell stage tooth } \\
\text { germ (embryonic } \\
\text { day }{ }^{19)}\end{array}$} & $\begin{array}{l}\text { Early Preameloblasts } \\
\text { (inner enamel epithelium near by cervical loop) }\end{array}$ & - & \pm & 1 & ++ \\
\hline & $\begin{array}{l}\text { Preameloblasts } \\
\text { (inner enamel epithelium adjacent to the predentin) }\end{array}$ & + & \pm & + & ++ \\
\hline & $\begin{array}{l}\text { Early preodontoblasts } \\
\text { (dental papilla cells near by cervical loop) }\end{array}$ & ++ & \pm & \pm & + \\
\hline & $\begin{array}{l}\text { Preodontoblasts } \\
\text { (dental papilla cells adjacent to the predentin) }\end{array}$ & + & + & + & ++ \\
\hline \multirow{5}{*}{$\begin{array}{l}\text { Late bell stage tooth } \\
\text { germ ( } 10,15 \text { days } \\
\text { after birth) }\end{array}$} & Secretory stage ameloblasts & \pm & \pm & ++ & ++ \\
\hline & Maturation stage ameloblasts & - & \pm & + & + \\
\hline & Early preodontoblasts & ++ & \pm & \pm & + \\
\hline & Preodontoblasts & + & + & + & ++ \\
\hline & Odontoblasts & \pm & ++ & ++ & ++ \\
\hline
\end{tabular}

++, Strong immunoreactivity; +, moderate immunoreactivity; \pm , weak immunoreactivity; -, negative immunoreactivity.

preodontoblasts were distributed in the dental papilla immediately below the epithelium (Fig. 1-a). An anti-LEF1 antibody yielded a positive reaction in preameloblasts. The reaction was not observed in the early preameloblasts of the cervical loop (Fig.1-b). Anti-P-Smad1/5/8 and anti-SP6 antibodies generated a uniform positive reaction in the inner enamel epithelium (Fig.1$\mathrm{d}$,-e). However, the reaction of anti-DKK1 antibody was weak in the inner enamel epithelium (Fig.1-c). In the dental papilla cells immediately below the inner enamel epithelium, the antibody reaction varied with regions. Specifically, anti-LEF1 antibody showed a strong positive reaction in early preodontoblasts adjacent to the cervical loop. However, the reaction was attenuated in preodontoblasts (Fig.1-b). In contrast, antiDKK1, anti-SP6, and anti-P-Smad1/5/8 antibodies yielded stronger reactions with preodontoblast differentiation from early preodontoblasts (Fig.1-c,-d,-e) (Table 1). LEF1 and P-Smad1/5/8 transcription factors generated strong immune reactions in the nuclei of positive cells (Fig.1-b,-e).

\section{Rat maxillary first molar germ 10 days after birth (late bell stage: crown formation)}

At the edge of tooth neck formation, secretory ameloblasts, odontoblasts, preodontoblasts adjacent to the predentin, and early preodontoblasts adjacent to Hertwig's epithelial root sheath were observed (Fig.2-a). Anti-DKK1 antibody yielded a strong positive reaction in odontoblasts (Fig.2-c). Anti-P-Smad1/5/8 and antiSP6 antibodies showed a strong positive reaction in secretory ameloblasts and odontoblasts (Fig.2-d,-e). However, these three antibodies yielded an attenuated reaction with the transition from odontoblasts to early preodontoblasts (Fig.2-c,-d,-e). In contrast, LEF 1 showed a strong reaction in the nucleus of early preodontoblasts, an attenuated reaction in preodontoblasts, and an unclear reaction in ameloblasts and odontoblasts (Fig.2-b) (Table 1).

\section{Rat maxillary first molar germ 15 days after birth (late bell stage: root formation)}

Mature ameloblasts and odontoblasts were observed around the cusp. Preodontoblasts were observed along predentin at the apical end of root formation. Early preodontoblasts were observed along Hertwig's epithelial root sheath (Fig.3-a). LEF1 yielded a strong positive reaction in the nuclei of early preodontoblasts. The reaction was attenuated with odontoblast differentiation from early preodontoblasts (Fig.3-b). In contrast, Dkk1, SP6, and PSmad1/5/8 showed a strong reaction in odontoblasts. These reactions were enhanced with odontoblast differentiation from early preodontoblasts (Fig.3-c,-d,-e). Mature ameloblasts were positive for SP6 and P-Smad1/5/8. Dkk1 yielded an unclear reaction, while LEF1 generated no reaction (Fig.3-b,-c,-d,-e,-f) (Table 1).

\section{Discussion}

Distributions of LEF1, DKK1, SP6, and p-Smad1/5/8 in odontoblasts and progenitor cells

Wnt-10a is involved in odontoblast differentiation through 
J.Hard Tissue Biology Vol. 22(4):481-488, 2013

the $\mathrm{Wnt} / \mathrm{B}$-catenin signaling pathway and in dentinogenesis and mineralization through DSPP induction ${ }^{3}$. However, LEF1 that acts downstream of the signaling pathway controls odontoblast differentiation by regulating DSPP ${ }^{6}$. LEF 1 is expressed in preodontoblasts and the dental papilla cells, which correspond to progenitor cells of preodontoblasts ${ }^{7)}$, but is not expressed in odontoblasts $^{8}$. Also, in the present experiment, the LEF1 reaction was adjacent to the cervical loop in tooth germs in the early bell stage and was increased in early preodontoblasts, lined by Hertwig's epithelial sheath, in the tooth germs in the late bell stage, but was decreased in preodontoblasts and became obscure in odontoblasts. These results suggest that LEF1 controls odontoblast differentiation and that odontoblasts appear as LEF1 disappears.

So, why does LEF1 not appear downstream of the Wnt/ $\beta$ catenin signaling pathway in odontoblasts, although Wnt-10a does appear upstream? One possible explanation is the involvement DKK. DKK1 is an inhibitor of the signaling pathway through binding to the Wnt receptor. DKK1 inhibits this pathway even in the presence of the Wnt signal ${ }^{19)}$. DKK1 prevents the stabilization of Wnt-induced $\beta$-catenin to reduce the transcriptional activity of Lef1 ${ }^{20)}$. DKK1 suppresses the appearance of Lef1 regardless of the appearance of Wnt-10a. Dkk1 is expressed in preodontoblasts and odontoblasts in tooth germs in the early bell stage before and after birth, but is not expressed in the dental papilla cells adjacent to the inner enamel epithelium in the cap stage $^{21)}$. We observed the tooth germs from the initial (cell differentiation) to late (hard tissue formation) bell stages. DKK1 was positive in dental papilla cells, which correspond to early preodontoblasts and preodontoblasts, in the early bell stage and in early preodontoblasts, preodontoblasts, and odontoblasts in the late bell stage. The positive reaction tended to be stronger with odontoblast differentiation. This finding of DKK1 is in contrast to that of LEF1. Specifically, DKK1 was replaced by LEF1, while, the reaction of LEF1 was attenuated with differentiation. Thus, LEF1, which controls odontoblast differentiation, may have disappeared through inhibition of the Wnt/ß-catenin signaling pathway mediated by DKK1.

In Sp6-deficient mice, Dkk1 decreased in the mandibular mesenchyme ${ }^{16)}$, in contrast, Lefl expression increased in the molar mesenchyme ${ }^{9}$. This suggests that SP6 reduction decreases DKK1, upregulating the Wnt signaling pathway and increasing LEF1. Sp6 is expressed in differentiated odontoblasts, but is not expressed in the dental mesenchyme in earlier stages ${ }^{15}$. Our results were consistent with those previously reported. Specifically, SP6 expression was weak in preodontoblasts in the early stages and tended to be stronger with odontoblast differentiation. This reaction tendency of SP6 was the same as that of DKK1 and in contrast to that of LEF1. Thus, besides DKK1, SP6 was closely involved in the disappearance of LEF1 in odontoblasts.
In the dental mesenchyme, Bmp4 induces Lef1 ${ }^{22}$. In mesenchymal cells of the cranial neural crest, the disappearance of Smad4, a transcription factor in the BMP signaling pathway, reduces Dkk1 and upregulates the Wnt signaling pathway ${ }^{23)}$. SP6 controls the BMP and Wnt signals during tooth development. In the dental papilla of Sp6-deficient mice, BMP is reduced ${ }^{24)}$. In the present study, we examined pSmad1/5/8, which activates the BMP signaling pathway, demonstrating a positive reaction in dental papilla cells immediately below the inner enamel epithelium and early preodontoblasts and a stronger positive reaction in preodontoblasts and odontoblasts. Thus, the BMP signaling pathway was activated with odontoblast differentiation. However, Bmp4 is strongly expressed in early preodontoblasts and is sharply decreased after differentiation, while Bmp 2 and 7 are strongly expressed in differentiated odontoblasts ${ }^{25,26,}$. Thus, pSmad1/5/8 activated the signaling pathway through BMP4 in preodontoblasts and through BMP2 and 7 in odontoblasts. Besides p-Smad1/5/8, LEF1 was strongly positive in early preodontoblasts with Bmp4 expression, suggesting that the BMP4 signaling pathway, activated through Smad1/5/8, was involved in LEF1 induction. Besides p-Smad1/5/8, SP6 and DKK1 were strongly positive in differentiated odontoblasts, while LEF1 was weakly positive. There were no effects on BMP4 in Sp6-overexpressing pulp cells ${ }^{24}$. SP6 that is strongly positive in differentiated odontoblasts may be involved in the activation of BMP2 and 7 through $\mathrm{p}-\mathrm{Smad} 1 / 5 / 8$.

\section{Distributions of LEF1, Dkk1, SP6, and p-Smad1/5/8 in ameloblasts and progenitor cells}

Lef1 is expressed in the inner enamel epithelium of early tooth germs $^{7)}$. Subsequently, lef1 disappears and is not expressed in ameloblasts $^{8)}$. Lef1 is involved in maintaining the precursor phenotype of the dental epithelium and is also employed as a marker of preameloblasts ${ }^{9}$. Also, in the present study, the inner enamel epithelium adjacent to predentin (i.e., preameloblasts) was reaction-positive. The reaction became weak in differentiated secretory ameloblasts, and disappeared in mature ones. Therefore, LEF1 decreased with ameloblast differentiation.

Sp6 is expressed in the inner enamel epithelium, including cervical loop, and preameloblasts and mature ameloblasts ${ }^{13,15}$. The overexpression of Sp6 in preameloblasts increases ameloblastin and decreases Lef ${ }^{19}$. Thus, SP6 promotes ameloblast differentiation from preameloblasts ${ }^{13}$. Also, in the present study, SP6 was expressed in the inner enamel epithelium, preameloblasts, and ameloblasts, and was increased in secretory ameloblasts. LEF1 decreased, while SP6 increased with ameloblast differentiation, suggesting that SP6 was involved in the decrease of LEF1 during ameloblast differentiation. SP6 promotes amelogenesis in vitro by regulating the expression of follistatin, an antagonist that inhibits receptor binding in the BMP signaling pathway ${ }^{13)}$. SP6 mediates the signaling pathway between Wnt/ß- 
Mitsuko Moriguchi et al.: LEF1, SP6, p-Smad1/5/8 and DKK1 in Developing Molars

catenin and $\mathrm{BMP}^{14)}$. We examined $\mathrm{pSmad} 1 / 5 / 8$, which activates the BMP signaling pathway, demonstrating a strong positive reaction in ameloblasts, except for mature ones, and inner enamel epithelium, suggesting that the BMP signaling pathway was activated with ameloblast differentiation and secretory functions. Bmp4 and 5 are expressed in the inner enamel epithelium in the bell stage and functional ameloblasts ${ }^{25,27)}$. Particularly, Bmp4 is strongly expressed in differentiating ameloblasts ${ }^{27}$. An antisense oligodeoxynucleotide to Bmp4 inhibits the differentiation of the inner enamel epithelium ${ }^{28}$. Thus, BMP4 and 5 are involved in ameloblast differentiation. BMP2 accelerates amelogenesis in vitro by activating Smad1/5/8 and increasing the expressions of Bmp4 and amelogenin, a marker of ameloblast differentiation ${ }^{29)}$. However, Bmp2 is weakly expressed in vivo in the inner enamel epithelium in the bell stage and ameloblasts ${ }^{27)}$. Therefore, pSmad1/ 5/8 activated the signaling pathway through BMP4 and 5, being involved in ameloblast differentiation. Sp6 is expressed in the fetal ectoderm under the control of the $\mathrm{Wnt} / \mathrm{B}$-catenin signaling pathway. The expression of Bmp4 is decreased in Sp6-deficient mice $^{14)}$. In the present study, SP6 was more strongly expressed in secretory ameloblasts than the inner enamel epithelium. This is consistent with the expression pattern of Bmp4 in the bell stage ${ }^{27}$. So, SP6 mediates the signaling pathway between Wnt/â-catenin and BMP4, being involved in ameloblast differentiation. Dkk1 is expressed in odontoblasts, but is not expressed in the inner enamel epithelium and ameloblasts ${ }^{21}$. In the present study, the immune reaction was unclear in both the inner enamel epithelium and ameloblasts, suggesting that DKK1 was not involved in ameloblast differentiation.

In summary, our findings suggest that, in ameloblasts and odontoblasts, LEF1 suppresses the differentiation into these cells, the activated BMP signaling pathway by $\mathrm{p}-\mathrm{Smad} 1 / 5 / 8$ suppresses the expression of LEF1 through SP6, and the downregulation of the Wnt signaling pathway by DKK1 is involved in the disappearance of LEF1 in odontoblasts. As a result, it is considered that the disappearance of LEF1 through SP 6 and p-Smad1/5/8 may be involved in the differentiation into odontoblasts and ameloblasts and their secretory functions.

\section{References}

1. Logan CY and Nusse R. The Wnt signaling pathway in development and disease. Annu Rev Cell Dev Biol 20: 781-810, 2004

2. Tolwinski NS, Wehrli M, Rives A, Erdeniz N, DiNardo $\mathrm{S}$ and Wieschaus E. Wg/Wnt signal can be transmitted through arrow/LRP5,6 and Axin independently of Zw3/Gsk3beta activity. Dev Cell 4; 407-418, 2003

3. Yamashiro T, Zheng L, Shitaku Y, Saito M, Tsubakimoto T, Takada K, Takano-Yamamoto T and
Thesleff I. Wnt10a regulates dentin sialophosphoprotein mRNA expression and possibly links odontoblast differentiation and tooth morphogenesis. Differentiation 75: 452-462, 2007

4. Sarkar L, Sharpe PT. Sarkar L and Sharpe PT. Expression of Wnt signalling pathway genes during tooth development. Mech Dev 85:197-200, 1999

5. Moriguchi M, Yamada M, Miake Y and Nitta Y. Immunolocalization of the factors related to Wnt signaling pathway in developing rat molar. J Hard Tissue Biology 20: 185-194, 2011

6. Yokose $\mathrm{S}$ and Naka T. Lymphocyte enhancer-binding factor 1: an essential factor in odontoblastic differentiation of dental pulp cells enzymatically isolated from rat incisors. J Bone Miner Metab 28: 650-658, 2010

7. Sasaki T, Ito Y, Xu X, Han J, Bringas P Jr, Maeda T, Slavkin HC, Grosschedl R and Chai Y. LEF1 is a critical epithelial survival factor during tooth morphogenesis. Dev Biol 278: 130-143, 2005

8. Sasaki H, Muramatsu T, Kwon HJ, Yamamoto H, Hashimoto S, Jung HS and Shimono M. Downregulated genes in mouse dental papillae and pulp. J Dent Res 89: 679-683, 2010

9. Nakamura T, de Vega S, Fukumoto S, Jimenez L, Unda $\mathrm{F}$ and Yamada Y. Transcription factor epiprofin is essential for tooth morphogenesis by regulating epithelial cell fate and tooth number. J Biol Chem 283: 4825-4833, 2008

10. Bafico A, Liu G, Yaniv A, Gazit A and Aaronson SA. Novel mechanism of Wnt signaling inhibition mediated by Dickkopf-1 interaction with LRP6/ Arrow. Nat Cell Biol 3: 683-686, 2001

11. Li L, Mao J, Sun L, Liu W and Wu D. Second cysteine-rich domain of Dickkopf-2 activates canonical Wnt signaling pathway via LRP-6 independently of dishevelled. J Biol Chem 277: 59775981, 2002

12. Fjeld K, Kettunen P, Furmanek T, Kvinnsland IH and Luukko K. Dynamic expression of Wnt signalingrelated Dickkopf1, -2 , and -3 mRNAs in the developing mouse tooth. Dev Dyn 233: 161-166, 2005

13. Ruspita I, Miyoshi K, Muto T, Abe K, Horiguchi T and Noma T. Sp6 downregulation of follistatin gene expression in ameloblasts. J Med Invest 55: 87-98, 2008

14. Talamillo A, Delgado I, Nakamura T, de-Vega S, Yoshitomi Y, Unda F, Birchmeier W, Yamada Y and Ros MA. Role of Epiprofin, a zinc-finger transcription factor, in limb development. Dev Biol 337: 363-374, 
2010

15. Nakamura T, Unda F, de-Vega S, Vilaxa A, Fukumoto S, Yamada KM and Yamada Y. The Krüppel-like factor epiprofin is expressed by epithelium of developing teeth, hair follicles, and limb buds and promotes cell proliferation. J Biol Chem 279: 626634,2004

16. Jimenez-Rojo L, Ibarretxe G, Aurrekoetxea M, de Vega S, Nakamura T, Yamada Y and Unda F. Epiprofin/ Sp6: a new player in the regulation of tooth development. Histol Histopathol 25: 1621-1630, 2010

17. Scott JE and Kyffin TW. Demineralization in organic solvents by alkylammonium salts of ethylenediaminetetra-acetic acid. Biochem J 169: 697-701, 1978

18. Adams JC. Heavy metal intensification of DABbased HRP reaction product. J Histochem Cytochem 29: 775,1981

19. Bafico A, Liu G, Yaniv A, Gazit A and Aaronson SA. Novel mechanism of Wnt signalling inhibition mediated by Dickkopf-1 interaction with LRP6/ Arrow. Nat Cell Biol 3: 683-6, 2001

20. Qiang YW, Barlogie B, Rudikoff S and Shaughnessy JD Jr. Dkk1-induced inhibition of Wnt signaling in osteoblast differentiation is an underlying mechanism of bone loss in multiple myeloma. Bone 42: 669-680, 2008

21. Fjeld K, Kettunen P, Furmanek T, Kvinnsland IH and Luukko K. Dynamic expression of Wnt signalingrelated Dickkopf $1,-2$, and -3 mRNAs in the developing mouse tooth. Dev Dyn 233: 161-166, 2005

22. Kratochwil K, Dull M, Farinas I, Galceran J and Grosschedl R. Lef1 expression is activated by BMP4 and regulates inductive tissue interactions in tooth and hair development. Genes Dev 10: 1382-1394, 1996
23. Li J, Huang X, Xu X, Mayo J, Bringas P Jr, Jiang R, Wang S and Chai Y. SMAD4-mediated WNT signaling controls the fate of cranial neural crest cells during tooth morphogenesis. Development. 138: 1977-1989, 2011

24. Ibarretxe G, Aurrekoetxea M, Crende O, Badiola I, Jimenez-Rojo L, Nakamura T, Yamada Y and Unda F. Epiprofin/Sp6 regulates Wnt-BMP signaling and the establishment of cellular junctions during the bell stage of tooth development. Cell Tissue Res. 350: 95-107, 2012

25. Bègue-Kirn C, Smith AJ, Loriot M, Kupferle C, Ruch JV and Lesot H. Comparative analysis of TGF beta s, BMPs, IGF1, msxs, fibronectin, osteonectin and bone sialoprotein gene expression during normal and in vitro-induced odontoblast differentiation. Int $\mathrm{J}$ Dev Biol. 38: 405-420, 1994

26. Yamashiro T and Tummers M. Thesleff I. Expression of bone morphogenetic proteins and Msx genes during root formation. J Dent Res 82: 172-176 2003

27. Aberg T, Wozney J and Thesleff I. Expression patterns of bone morphogenetic proteins (Bmps) in the developing mouse tooth suggest roles in morphogenesis and cell differentiation. Dev Dyn 210: 383-396, 1997

28. Tabata MJ, Fujii T, Liu JG, Ohmori T, Abe M, Wakisaka S, Iwamoto M and Kurisu K. Bone morphogenetic protein 4 is involved in cusp formation in molar tooth germ of mice. Eur J Oral Sci 110:114120, 2002

29. Miyoshi K, Nagata H, Horiguchi T, Abe K, Arie Wahyudi I, Baba Y, Harada H and Noma T. BMP2induced gene profiling in dental epithelial cell line. J Med Invest 55: 216-226, 2008 